livraisons

d'Histoire

de l'Architecture

\section{Livraisons de l'histoire de l'architecture}

$40 \mid 2020$

À propos des princes de Conti

\title{
À propos des « copies de musique faites par J.J. Rousseau pour S.A.S. Monseigneur le prince de Conti »
}

À propos des « copies de musique faites par J.J. Rousseau pour S.A.S.

Monseigneur le prince de Conti »

Regarding "copies of music made by J.J. Rousseau for H.S.H Monsignor the Prince de Conti"

Anmerkungen zu den "Copies de musique faites par J.J. Rousseau pour S.A.S.

Monseigneur le prince de Conti"

Thomas Vernet

(2) OpenEdition

Journals

Édition électronique

URL : http://journals.openedition.org//ha/1287

DOI : 10.4000/lha. 1287

ISSN : 1960-5994

Éditeur

Association Livraisons d'histoire de l'architecture - LHA

Édition imprimée

Date de publication : 3 décembre 2020

Pagination : $33-44$

ISSN : $1627-4970$

Référence électronique

Thomas Vernet, «À propos des « copies de musique faites par J.J. Rousseau pour S.A.S.

Monseigneur le prince de Conti » », Livraisons de l'histoire de l'architecture [En ligne], 40 | 2020, mis en ligne le 28 décembre 2020, consulté le 24 mars 2021. URL : http://journals.openedition.org//ha/1287 ; DOI : https://doi.org/10.4000/lha.1287 


\section{À PROPOS DES «COPIES DE MUSIQUE FAITES PAR J.-J. ROUSSEAU POUR S.A.S. MONSEIGNEUR LE PRINCE DE CONTI»}

Le copiste musical que fut Jean-Jacques Rousseau a déjà fait l'objet de plusieurs études, depuis l'ouvrage ancien mais toujours fondamental d'Albert Jansen, Rousseau als Musiker jusqu'au plus récent article de Christine Waeber ${ }^{1}$. Toutefois un mémoire autographe, conservé à la Bibliothèque publique et universitaire de Neuchâtel, intitulé Copies de Musique faites par J.-J. Rousseau pour S.A.S. Monseigneur le Prince de Conti ${ }^{2}$ semble avoir encore échappé à l'attention des historiens et des musicologues. C'est sur cette pièce d'archive que se fonde notre recherche, dont nous présentons ici un premier état. Celle-ci vise à enrichir la connaissance de l'activité de copiste du " citoyen de Genève » en même temps que celle du foyer musical du palais prieural du Temple à Paris, où le prince du sang établi un concert prestigieux dès le début des années 1760 .

Le document, dont nous proposons une transcription en annexe, se présente à la manière d'un mémoire comptable. Il recense les titres de quarante-quatre œuvres de musique vocale italienne réparties sous deux rubriques précédées d'une lettre majuscule - D. pour les airs et ensembles ("Duetto", "terzetto") et E. pour les récitatifs et airs. À l'intérieur de ces deux sections, chaque pièce est désignée par son incipit littéraire, sans mention de compositeur, rendant l'identification peu aisée. Cette présentation n'est pas sans lien avec celle que Rousseau adoptera, à partir du $1^{\text {er }}$ avril 1772, dans le catalogue de ses travaux de copie musicale:

Chaque pièce grande ou petite, copiée soit en partition soit en parties séparées, a son numéro dans l'ordre où elles ont été faites et sous chacun de ces numéros est marquée la date du jour où la copie a été achevée, le nom de la personne pour qui elle a été faite, ensuite l'indication de la pièce, le nombre

1. Albert Jansen, Rousseau als Musiker, Berlin, Reimer, 1884, reprint Genève, 1971, 482 p. ; Jacqueline Waeber, "Rousseau copiste de musique : l'envers de l'auteur ?", Jean-Jacques Rousseau en 2012, "Puisqu'enfin mon nom doit vivre", dir. Michael O’Dea, Oxford, Voltaire foundation, 2012, p. 197-222 ; on pourra également consulter sur le sujet: Ellen S. Burt, "Rousseau The scribe ", Studies in romantism, $18: 4$ (1979), p. 629-667 ; Béatrice Didier, La Musique des Lumières, Paris, Presses universitaires de France, 1985, 480 p. ; Catherine Kintzler, Poétique de l'opéra français de Corneille à Rousseau, Paris, Minerve, 1991, 486 p.; Jean-Jacques Eigeldinger (dir.), Jean-Jacques Rousseau et la musique, À la Baconnière, 1988, 140 p.; Cécile Reynaud, "Jean-Jacques Rousseau, compositeur et copiste ", Revue de la Bibliothèque nationale de France, no 42, 2012, p. 81-88.

2. Bibliothèque publique et universitaire de Neuchâtel, MsR-NA9 fol ${ }^{\circ} 4-7$, Copies de Musique//pour S.A.S. Monseigneur le Prince de Conti//par J.-J. Rousseau. 
des parties séparées quand il y en a, et enfin le nombre des pages, avec la distribution de celles qui sont en grand format, et qui contiennent souvent le double des autres. Pour ne pas trop multiplier les numéros, j'en ai borné le nombre à cent, après quoi je recommence une seconde centaine et je distingue les centaines par autant de lettre de l'Alphabet, donnant l'A à la première centaine, le $\mathrm{B}$ à la suite ${ }^{3}$.

On aurait aimé voir figurer sur le mémoire de Neufchâtel les dates d'achèvement des copies, le nombre de parties séparées réalisées le cas échéant, s’il s'agissait de grandes partitions ou de partitions réduites... Mais sans doute ces détails n'avaient-ils pas leur place sur un document comptable dont l'objet principal était de justifier au commanditaire le prix réclamé pour la réalisation de chaque partition copiée, fixé selon le nombre de pages noircies. En l'espèce, le corpus désigné ici comprend quatre-cent-trente-trois et demi, représentant montant total de cent vingtsix livres et quatre-vingt-quinze sols.

\section{Jean-Jacques copiste musical...}

C'est probablement en septembre 1731, alors qu'il séjournait à Lyon, que Rousseau commença à exercer professionnellement le métier de copiste de musique ; activité qu'il poursuivit au moins jusqu'à l'été 1777. Au milieu du XVIII ${ }^{\mathrm{e}}$ siècle, alors que seule l'imprimerie permettait de reproduire mécaniquement un texte, la profession de copiste de musique n'avait rien perdu de sa légitimité établie de longue date. Toutefois, Rousseau fut le premier, dans son Dictionnaire de musique publié en décembre 1767, à donner, dans un article d'une ampleur inhabituelle, une définition lexicographique du terme "copiste" dans un contexte purement musical ${ }^{4}$. Rappelons que la première édition du Dictionnaire de l'Académie française (1694) comprenait bien une définition du "copiste " mais sans allusion à sa fonction dans le domaine de la musique ${ }^{5}$; il faudra pour cela attendre sa sixième édition $(1835)^{6}$. De même Furetière $(1690)$ consacre trois entrées au "copiste " mais sans qu'aucune n'évoque le professionnel qui se consacre à reproduire de sa main des partitions à des fins commerciales ${ }^{7}$. Même Sébastien de Brossard qui avait lui-

3. Albert Jansen, Rousseau als Musiker, op. cit., p. 474 ; la localisation de ce document n'est plus connue aujourd'hui.

4. Jean-Jacques Rousseau, Dictionnaire de musique, fac-similé de l'édition de 1768, éd. préparée et présentée par Claude Dauphin, Arles, Actes Sud, coll. Thesaurus, 2007, p. 124-132. L'article "Copiste " a été rédigé probablement aux alentours de l'année 1755.

5. Dictionnaire de l'Académie française (1694), voir The ARTFL Project, section "Dictionnaires d'autrefois », https://portail.atilf.fr/dictionnaires/ACADEMIE/PREMIERE/premiere.fr.html [consulté le 17 juin 2020].

6. Id., https://portail.atilf.fr/dictionnaires/ACADEMIE/SIXIEME/sixieme.fr.html [consulté le 17 juin 2020].

7. Antoine Furetière, Dictionnaire universel (1690), voir http://www.xn--furetire-60a.eu/index.php/ non-classifie/709739159- [consulté le 17 juin 2020]. 
même tant copié de musique et offert avec son propre Dictionnaire de musique - soixante-quatre ans avant celui de Rousseau - le premier glossaire musical francoitalien, ne donne pas non plus d'entrée pour ce terme ${ }^{8}$. Et si l'Encyclopédie de Diderot et d'Alembert utilise l'expression à deux reprises dans un contexte musical, celle-ci était fréquemment en usage depuis au moins la première moitié du siècle 9 .

La popularité grandissante des maisons d'opéra en Europe et le rayonnement de la musique italienne ne firent que favoriser l'emploi de copistes. Dans son article, Rousseau exagère à peine lorsqu'il écrit "qu'en Italie, le pays de la terre où l'on fait le plus de Musique, on a proscrit depuis longtems la Note imprimée sans que l'usage de la gravure ait pu s'y établir ${ }^{10}$ ». Les copies auxquelles Rousseau fait ici référence ne sont pas celles réalisées à des fins d'archivages ou patrimoniales, mais pour favoriser la circulation du répertoire parmi les musiciens. Leur fonction n'était pas de fixer définitivement le texte musical, puisqu'il s'agissait de partitions d'usage ; d'où leurs lacunes fréquentes - signes de la liberté interprétative laissée aux chanteurs ou aux instrumentistes et au statut de la partition, considérée d'abord comme un aide-mémoire. Lors de son séjour vénitien en 1744-1745, Rousseau avait vraisemblablement eu entre les mains ces partitions d'opéras qui circulaient exclusivement sous la forme de manuscrits de copistes. Si des ateliers étaient attachés à des institutions lyriques, il n'était pas rare que des copistes exercent leur métier de manière indépendante. Il s'agissait le plus souvent de maîtres de musique pour lesquels la copie représentait un complément de revenu salutaire. Certains iront jusqu'à se spécialiser dans un domaine précis - musique instrumentale, répertoire de l'opéra italien ou celui de l'opéra-comique... L'activité de copiste de Rousseau s'inscrit dans cette catégorie, celle des indépendants travaillant "à la pièce ".

En 1750, à la suite d'ennuis de santé, Rousseau décida de trouver un moyen de subsistance indépendant: "J'en imaginai un moyen très simple ce fut de copier de la musique à tant la page... de caissier d'un financier je me fis copiste de musique ${ }^{11}$ ".

Le Registre de mes copies de musique commencé le $1^{\text {er }}$ avril 1772 déjà mentionné, qui recense les copies qu'il effectua depuis le $1^{\text {er }}$ septembre 1770 illustre bien cette pratique. On se souvient que dans le Deuxième dialogue de Rousseau juge de JeanJacques, il indiquait avoir écrit "dans ces six ans [1772-1777] [...] en simple copie plus de six mille pages, dont une partie, musique de harpe et de clavecin, ou solo et concerto de violon très chargée et en plus grand papier, qui demande une grande attention et prend un temps considérable ${ }^{12}$ ». Le département de la Musique de la Bibliothèque nationale de France conserve le plus important ensemble de manuscrits autographes d'œuvres musicales de Rousseau réuni dans ce que l'on a coutume

8. Sébastien de Brossard, Dictionnaire de musique, contenant une explication des termes grecs, latins, italiens et françois le plus usitez dans la musique..., Paris, C. Ballard, 1703.

9. Voir Jacqueline Waeber, "Rousseau copiste de musique : l'envers de l'auteur ? ", op. cit., p. 200-201.

10. Jean-Jacques Rousseau, Dictionnaire de musique, op. cit., p. 124.

11. Jean-Jacques Rousseau, Les Confessions, Paris, Garnier, 1923, Livre VIII, p. 337.

12. Jean-Jacques Rousseau, Rousseau juge de Jean-Jacques, $2^{\mathrm{e}}$ dialogue, cité par Arthur Pougin, JeanJacques Rousseau musicien, Paris, Fischbacher, 1901, p. 44. 
d'appeler le "recueil Benoît », du nom de Pierre-Antoine Benoît (1721 ?-1796 ?), son déposant à la Bibliothèque royale dès le 10 avril 1781. Ce fort volume de 601 pages e musique regroupe 132 pièces essentiellement vocales de genre divers - chansons, ariettes, duos, fragments de Daphnis et Chloé, de motets latins - et de quelques pièces instrumentales. Ce vaste corpus avait été trouvé dans les papiers laissés par Rousseau au lendemain de sa mort, le 2 juillet 1778, chez le marquis de Girardin à Ermenonville ${ }^{13}$.

Cependant, ce registre n'est pas pleinement représentatif de ce que fut la carrière du copiste Rousseau. En effet, il ne recouvre qu'une petite portion chronologique de son activité et révèle surtout qu'à la fin de sa vie, Rousseau recopia principalement sa propre musique: chansons, romances, airs tirés de ses ouvrages - Le Devin $d u$ village et Daphnis et Chloé. Il y a certes ici une référence à une copie de l'Olimpiade de Pergolesi mais le répertoire italien qu'il avait tant affectionné y est presqu'absent. Plusieurs autres dons et acquisitions ultérieurs complètent le fonds de la BnF : le legs Malherbe a apporté en 1912 à la bibliothèque du Conservatoire, la partition du motet Ecce sedes hic tonantis ${ }^{14}$, tandis que les papiers de la compositrice Sophie Gail (1775-1819), conservés dans cette même bibliothèque, contenaient les parties de chant du duo "Tant qu'à mon Colin j'ai seu plaire ", extrait des nouveaux airs du Devin du village ${ }^{15}$. Enfin en 1990, le département de la Musique de la BnF a acquis un recueil autographe de Canzoni italiennes de Rousseau - Raccolta di canzoni Veneziane e toscane, qui ne comporte aucune pièce commune avec le "recueil Benoît " et qui fut probablement réalisé avant que Rousseau ait commencé à rédiger ces inventaires de $1772^{16}$. Un autre témoignage de son activité de copiste, antérieurement à cette date tardive est fourni par la bibliothèque musicale du baron Grimm qui fut saisie sous la Terreur par le violoniste Bruni sur ordre de la Commission Temporaire des Arts. Cette collection étudiée par Catherine Massip, contenait un corpus de musique vocale italienne, parmi lesquelles figuraient 36 partitions copiées ou attribuées à la main de Rousseau ${ }^{17}$.

13. F-Pn., Res. $\mathrm{Vm}^{7}$ 667. Pour plus de détail sur le recueil Benoît, voir Cécile Reynaud, "Jean-Jacques Rousseau copiste et compositeur ", op. cit. La multiplication des attestations de ce dépôt émises par les proches de Rousseau souligne l'importance que cette démarche revêtait à leurs yeux.

14. F-Pc., Ms. 379 : Ecce sedes hic tonantis, motet pour voix seule et orchestre, composé pour la dédicace de la chapelle du château de Chevrette en 1757, avec les annotations et titres de l'auteur.

15. F-Pc., Ms. 380. Parties de chant seules, avec indications de ritournelles des violons.

16. F-Pn., Ms 21646.

17. Voir Catherine Massip, "La bibliothèque musicale du baron Grimm ", D’un opéra l'autre. Hommage à Jean Mongrédien, dir. Jean Gribenski, M.-C. Mussat et Herbert Schneider, Paris, Presses de l'Université de Paris-Sorbonne, 1996, p. 189-205. 


\section{... au service du prince de Conti}

On connaît depuis l'article éclairant de Jean Fabre, quelle fut la nature des rapports entretenus pendant une dizaine d'années entre Rousseau et Louis-François de Conti ${ }^{18}$. Le mémoire de Neuchâtel n'est pas daté mais se rapporte, comme on va le voir, aux premières années de leur relation (1759-1762) ; période mieux connue par l'anecdote des deux paniers de gibier "tué de sa main » que le prince fit porter à Rousseau ${ }^{19}$, celle du chien qu'il lui offrit pour remplacer le "pauvre Turc ${ }^{20}$, et plus encore par les deux visites que Conti rendit à Jean-Jacques en septembre 1760, que celui-ci considéra comme «le plus grand honneur que les lettres [lui] aient valu et auquel [il a] été le plus sensible ${ }^{21}$.

C'est par l'intermédiaire d'"éclaireurs » que Conti avait cherché à entrer en contact avec Rousseau en 1759, alors que celui-ci était l'hôte de Madeleine Angélique de Neufville et de son époux, le maréchal Charles-François de Luxembourg, à Montmorency. Le premier des émissaires du prince fut le chevalier de Lorenzi (1712-1784), gentilhomme d'origines florentines qui, à l'issue d'une carrière militaire dans les armées françaises (1733-1757) était devenu son secrétaire ${ }^{22}$. Après avoir fait la connaissance de Rousseau chez les Luxembourg, celui-ci devait cultiver une amitié sincère pour son "cher solitaire ", renforcée par une même connaissance de la littérature italienne et une égale passion pour le jeu d'échecs. Le chevalier de Lorenzi fut également le «sigisbée » de la comtesse Amélie de Boufflers (1724-1800), la maîtresse du prince de Conti, qui joua également un rôle d'émissaire auprès de Jean-Jacques. Celui-ci s'attacha lui-même à "l'idole du Temple " avant, comme à son habitude, de se défier de cette amitié " mixte ${ }^{23}$.

Au fil de la correspondance générale de Rousseau, il est possible de reconstituer les étapes de son travail de copiste au service du prince de Conti, qui s'inscrivit à la suite de celui réalisé pour le baron Grimm. Entre le 18 octobre et le 6 novembre 1760, Rousseau échange avec Lorenzi plusieurs lettres dans lesquelles il est question d'une commande de papier à musique pour honorer les commandes du prince ainsi que celle d'un " grand papier » possiblement destiné à la copie de la Nouvelle Héloïse :

18. Jean Fabre, "Jean-Jacques Rousseau et le prince de Conti ", Lumières et Romantisme. Énergie et nostalgie de Rousseau à Mickiewicz, 2e éd. revue et corrigée, Paris, Klincksieck, 1980, p. 101-135. Pour la chronologie de cette relation, voir Raymond Trousson et Frédéric S. Eigeldinger, JeanJacques Rousseau au jour le jour, Paris, Honoré Champion, 1998, 431 p.

19. 7 octobre et 6 novembre 1760 ; voir Raymond Trousson et Frédéric S. Eigeldinger, Jean-Jacques Roussean au jour le jour, op. cit., p. 129 et 131.

20. 21 juillet 1761 , ibid., p. 148.

21. Les dates exactes de ces deux visites que Conti rendit à Rousseau à Montmorency - au PetitChâteau pour la première et à Montlouis pour l'autre - ne sont pas connues; voir Euvres complètes, éd. Bernard Gagnebin, et Maurice Raymond, Paris, Gallimard, "Bibliothèque de la Pléiade ", 1959-1995, 56 vol., tome I, p. 542-543.

22. Sur Orlando de Lorenzy, voir Dictionnaire de Jean-Jacques Rousseau, dir. Raymond Trousson et Frédéric S. Eigeldinger, Paris, Honoré Champion, 2006, p. 565.

23. Voir Chantal Mustel, "Rousseau et "l'idole du Temple" ", Les Trésors des princes de Bourbon Conti, cat. Exp. L'Isle-Adam, musée d'Art et d'Histoire Louis-Senlecq, éd. Frédéric Chappey, Paris, Somogy Éditions d'art, 2000, p. 48-52. 
L'explication que vous m'avez donnée au sujet du papier ne vous justifie pas tout à fait de la profusion dont je vous accuse; mais comme j'aurai peu d'argent à débourser grace à l'attention de M. le Prince de Conti; je ne me plains pas beaucoup d'une dépense que je ne dois payer qu'en chansons. Afin donc de n'être pas chargé d'in dépôt, je prendrai le papier pour mon compte au moyen de quoi je taxerai ma copie comme si j'en avois fourni le papier, et nous déduirons sur le payement trente trois livres avancées par S.A. Quant à vous, je consens à ne vous rembourser les 9 francs qu'à nôtre première entrevüe; mais je voudrois bien ne les pas garder trop longtems.

Je dois vous dire que le grand papier destiné à la copie du manuscrit a été un peu limé par le dos dans la voiture, ce qui peut rendre la reliure plus difficile et moins solide; d'ailleurs la forme m'en paroit bien grande pour être employé dans toute sa grandeur. Ne conviendrait-il pas de le plier en deux pour lui donner un format in $4^{\circ}$ à peu près comme celui du manuscrit. De cette manière la limure ne seroit plus au dos, mais sur la tranche, et cela s'en iroit en le reliant. Vous pourrez là-dessus savoir à loisir les intentions du Prince ; car j’ai commencé par la musique, et je ne prendrai le manuscrit que quand elle sera faite ${ }^{24}$.

Puis du 21 novembre au 22 décembre, c'est la copie de l'air Se Perde il caro Bene, la fida Tortorella extrait de La Cameriera accorta de Baldassare Galuppi qui occupe notamment la Correspondance:

J'ay receu aussi la seconde Copie de l'Ariette de la Tortorella, et j'ay remis la premiere a Me Le P. De Conti qui m'a dit qu'il etoit bien aise de L'avoir aussi detaché, et qu'il etoit juste que vous m'envoyassiés le Compte de ce qu’il vous doit pour ce que Vous avés déjà copié, affin que Vous ne fussés pas en debourcé en attendant le reste du Livre que Vous n'aurés pas vraisemblablement Si tot fini ${ }^{25}$.

Puis, au mois de juin 1761 , on retrouve, cette fois sous la plume de $\mathrm{M}^{\text {me }} \mathrm{de}$ Boufflers, une allusion aux copies musicales attendues par le prince de Conti. Le 4 de ce mois, la comtesse en même temps qu'elle s'informe de la santé de Rousseau, souffrant au moins depuis le mois de mai, tient à le rassurer sur le fait que les commandes ne "pressent pas":

M. le Prince de Conti a su Monsieur, que vous aviez été incommodé ; il craint que vous ne vous fatiguiez en copiant la musique qu'il vous a donné, il m'a chargé de vous dire qu'il n'en est pas fort pressé maintenant, qu'il vous prie de ne vous en point tourmenter ${ }^{26}$.

24. Jean-Jacques Rousseau, Correspondance complète, éd. Ralph A. Leigh, Genève, Institut et Musée Voltaire, puis Oxford, The Voltaire Foundation, 1995-[1997], 51 vol., tome VIII, n ${ }^{\circ} 1142$, Rousseau au chevalier de Lorenzi, 3 novembre 1760, p. 280-282.

25. Ibid., $\mathrm{n}^{\circ}$ 1202, Chevalier de Lorenzi à Rousseau, 22 décembre 1760, p. 365-367. Cet air n'est pas mentionné dans le mémoire de Neuchâtel.

26. Ibid., t. IX, no 1425, $\mathrm{M}^{\mathrm{me}}$ de Boufflers à Rousseau, 4 juin 1761, p. 2. 
Un mois plus tard, le 12 juillet, $\mathrm{M}^{\text {me }}$ de Boufflers lui demande toujours de la part de Conti, le montant des copies effectuées à sa demande, mais souhaite surtout savoir s'il est vrai qu'il ne veut "plus rien copier" :

M. le Prince de Conty m'a chargé, Monsieur, de m'informer de ce qu'il vous doit, et s'il est vray que vous ne vouliez plus rien copier. Je vous prie de vouloir bien me repondre a ces deux questions ${ }^{27}$.

Enfin le 21 juillet, tandis que $\mathrm{M}^{\mathrm{me}}$ de Boufflers prend part au chagrin de Rousseau après la mort de son chien Turc et exprime les regrets du prince, elle accompagne sa lettre du règlement des sommes dues pour les copies : "Voila Monsieur les 196 lt $16 \mathrm{~s}$ qui vous sont dus qu'il m'a chargé de vous remettre ${ }^{28}$ ".

Il est possible que le mémoire de Neuchâtel renvoie à cette commande passée au début l'été 1761. Mais comment dans ce cas interpréter la différence entre la somme annoncée par $\mathrm{M}^{\mathrm{me}}$ de Boufflers et celle déduite du mémoire ? Celui-ci est-il incomplet? On pourrait le croire, la plume de Rousseau paraissant avoir été suspendue après le $14^{\mathrm{e}}$ item de la lettre E... Pour plus d'assurance, il conviendrait de pouvoir le comparer avec un autre compte que Rousseau aurait au prince de Conti et qui selon les fichiers Charavay ( $n^{\text {os }} 104-117$ et 320-324), passa dans deux ventes au XIX ${ }^{\mathrm{e}}$ siècle avant que l'on en perde ${ }^{29}$.

\section{Les copies musicales de Rousseau, reflets de l'activité de l'orchestre du prince?}

À défaut de pouvoir restituer avec certitude les airs recensés dans le mémoire de Neuchâtel à leur compositeur, il est néanmoins possible de formuler des hypothèses d'attributions grâce, notamment, à l'exploitation du Répertoire International des Sources Musicales ${ }^{30}$. Celle-ci fait apparaître la cohérence et l'unité du corpus copié par Rousseau à la demande de Conti. Celui-ci est composé en majorité d'airs séparés tirés d'ouvrages lyriques italiens composés en majorité entre la fin des années 1740 et le milieu de la décennie suivante. Mais en l'état de nos connaissances, il est difficile de pousser plus loin les investigations. Attendu que cette source consigne uniquement les incipits littéraires des airs copiés par Rousseau, il est bien hasardeux de les attribuer aux divers compositeurs qui, dans bien des cas ont mis les mêmes textes en musique; on pense en particulier aux airs composés sur des vers de Métastase $^{31}$.

27. Ibid., t. IX, no $1447, \mathrm{M}^{\text {me }}$ de Boufflers à Rousseau, 12 juillet, p. 59.

28. Ibid., t. IX, no 1455, $\mathrm{M}^{\mathrm{me}}$ de Boufflers à Rousseau, 21 juillet 1761, p. 68-69.

29. Ce document se présentait sous la forme de 4 pages, et faisait, curieusement, état de 44 morceaux dont tous les titres étaient indiqués copiés à raison de 6 sols la page, soit un total de 196 livres et 16 sols. C'est là une description bien proche de la physionomie du mémoire de Neuchâtel. Voir Jean-Jacques Rousseau, Correspondance complète, op. cit., t. IX, p. 59.

30. http://www.rism.info/home.html. Nos hypothèses d'attribution ont été intégrées entre crochets carrés à la transcription du mémoire de Neuchâtel proposée en annexe.

31. Pour une vue d'ensemble des opéras écrits sur des livrets de Métastase, on se référera à l'ouvrage d'Alfred Wotquenne, Alphabetisches Verzeichnis des Stücke in Versen aus den dramatischen Werken von Zeno, Metastasio und Goldoni, Leipzig, Breitkopf und Härtel, 1905, 77 p. 
Par exemple, le livret de son Demofoonte (1733), dont est tiré ici l'air « Non cura l'affetto" (D. no 25), a été mis en musique par au moins sept compositeurs, dont Rousseau aurait été susceptible de copier la partition...

Toutefois, en dépit de ces lacunes, on peut souligner qu'au moment où Conti passa ces commandes à Rousseau, le répertoire qui en faisait l'objet était encore relativement peu diffusé en France à travers l'édition et apparemment peu présent dans les programmes de concerts publics. Les premières années de la décennie 1760 correspondent par ailleurs à la période de constitution, au palais prieural du Temple et par la volonté même du prince, d'un prestigieux foyer musical, creuset des mutations esthétiques propres à favoriser l'émergence d'une nouvelle sensibilité ${ }^{32}$. Après le déclin du mécénat de La Pouplinière, chanteurs comme instrumentistes devaient trouver dans l'Enclos une nouvelle protection et c'est ainsi que l'orchestre du Temple passa rapidement pour «l'un des meilleurs et des plus complets qu'on [put] voir » ${ }^{33}$. Tandis que l'on assiste alors au déplacement des artistes d'un foyer musical à l'autre, les copies réalisées par Rousseau témoignent peut-être de la volonté de Conti de se constituer un "fonds de répertoire " susceptible d'alimenter ses concerts "à jour marqué ". Dans sa lettre du 21 novembre 1760, le chevalier de Lorenzi demandait à Rousseau de lui "envoyer cet air Se perde il caro bene la fida Tortorella que Mr. le Prince de Conti [l'avait] chargé de remettre de Sa part à Mdme St. Aubin, et qu'Elle [le] press[ait] de luy donner ${ }^{34}$ ". L'identité de cette "Mdme St. Aubin " n'est pas clairement établie mais on peut suggérer qu'elle désigne Jeanne-Thérèse Goërmans (1727- ?) qui se faisait justement appeler $\mathrm{M}^{\mathrm{me}}$ de Saint-Aubin depuis qu'elle avait été la maîtresse de La Pouplinière en 1735. Fille du facteur de clavecins parisien Jean Goërmans, elle avait gagné sa vie avant son mariage en donnant des leçons de musique et se produisant en concert. Après le second mariage du fermier général en juillet $1759, \mathrm{M}^{\mathrm{me}}$ de Saint-Aubin avait repris son ancien métier et on la retrouvait dans le monde. Dans un tel contexte, il n'est pas improbable que le prince de Conti se soit adressé indirectement à elle pour solliciter le prêt de certaines partitions dont il confia la réalisation des copies à Rousseau pour son propre orchestre...

Mais les relations musicales entre Conti et Rousseau ne se bornèrent pas seulement à ces seules commandes de copies d'œuvres de compositeurs étrangers. Le prince fut lui-même sensible à la musique de l'auteur du Devin du village. Ainsi leurs

32. Sur l'activité du foyer musical du Temple, voir Frédéric Bussmann, Un Prince collectionneur. LouisFrançois de Bourbon Conti et ses collections au palais du Temple à Paris, Éditions de la Maison des sciences de l'homme, Centre Allemand d'Histoire de l'art, 2012, p. 77-83; Gaston Capon et Robert Yves-Plessis, Paris galant au XVIII e siècle. Vie privée du prince de Conty, Louis-François de Bourbon (1717-1776), Paris, Schemit, 1907, p. 129-139; David Hennebelle, De Lully à Mozart. Aristocratie, musique et musiciens à Paris (XVII -XVIII $^{e}$ siècles), Seyssel, Champ Vallon, 2009, p. 236-244; Thomas Vernet, "Les collections musicales des princes de Conti ", Musique, Images, Instruments. Revue d'organologie et d'iconographie musicale, no 8, 2006, p. 44-67.

33. [Bouffonidor], Les Fastes de Louis XV, de ses ministres, maîtresses, généraux et autres notables personnages de son règne, Villefranche, $\mathrm{V}^{\mathrm{ve}}$ Liberté, 1785, 2 vol. de 4 tomes, t. I, p. LXXXVIII.

34. Jan-Jacques Rousseau, Correspondance complète, op. cit., t. VIII, no 1169 , Chevalier de Lorenzi à Rousseau, 21 novembre 1760 , p. 323. 
échanges de la fin de l'année 1761 tournèrent-ils notamment autour de la partition des Muses Galantes que le prince souhaita faire jouer à l'occasion des fêtes de fin d'année passées à L'Isle-Adam. Dans le même temps, il commanda à Rousseau « un petit drame lyrique» sur des paroles de son choix "pourvu, [avait-il fait savoir à Rousseau par l'intermédiaire de Lorenzi] qu'Il entende de cette Musique naïve et sensible qui L'enchante differemment de tout autre, et luy fait sentir un plaisir qu'Il n'éprouve pas dans toute autre Musique ${ }^{35}$ ». Mais l'histoire ne dit pas si le musicienphilosophe mena à bien ce projet. Lorsqu'il réapparut au Temple en janvier 1768, ses talents de compositeurs furent toutefois contestés :

M. Rousseau de Genève étant venu à Paris avec son opéra des Neuf Muses, que les nouveaux directeurs lui ont demandé, il s'en est fait une répétition chez le prince de Conti au Temple, où l'on a conclu que cet opéra n'étoit pas jouable ${ }^{36}$.

Et l'on ignore si son motet Quam dilecta tabernacula ${ }^{37}$, composé à Trie en 1767-1768, fut joué au Temple.

Les volumes qui formaient la bibliothèque musicale du prince de Conti ont malheureusement été perdus. L'inventaire dressé après le décès du prince laisse cependant apparaître une proportion importante de manuscrits et de partitions non reliés probablement destinés à la pratique qui purent constituer une partie du répertoire de l'orchestre du Temple.

883 - Item trente portefeuilles en Carton numérotés 242 contenant la Musique manuscritte [sic] de divertissement de différents operas dont celle des Indes Galantes prisés ensembles six livres.

884 - Item douze portefeuilles de musique Manuscrites numérotés 243 contenant des Simphonies de divers auteurs prisés ensembles six livres.

885 - Item huit portefeuilles en Carton de musique Manuscrites numérotés 244 contenant des duos et autres Musique Instrumentale prisés ensembles livres.

886 - Item trente six portefeuilles en carton de Musique Manuscrite numérotés 245 Contenant les partitions de différents opéras comiques prisés ensembles dix-huit livres.

887 - Item vingt trois volumes in quarto de différentes Musiques gravées numérotés 246 dont pièces de Clavecin d'Andrieu [sic] prisés ensembles six livres ${ }^{38}$.

35. Ibid., t. IX, $\mathrm{n}^{\circ}$ 1549, Chevalier de Lorenzi à Rousseau, 18 novembre 1761, p. 247-248.

36. Mémoires secrets pour servir à l'histoire de la république des Lettres en France, depuis 1762 jusqu’à nos jours, dir. Christophe Cave et Suzanne Cornand, Paris, Honoré Champion, 2009, 15 janvier 1768, vol. II, p. 845 ; voir aussi Édouard-Georges Jacques Grégoire, Les Gloires de l'Opéra et la musique à Paris, Bruxelles, Paris, Londres, Schott frères, 1881, 3 vol., t. III, p. 338.

37. F-Pn., Res. $\mathrm{Vm}^{7}$ 667, fol. 383-295: «Mottet à deux voix pour Madame de Nadaillac abbesse de Gomerfontaine qui a fourni les paroles ».

38. Arch. nat., $\mathrm{X}^{1}$ A 9178 et 9179 , Scellés et inventaires après décès de Louis-François de Conti, 1776. 
Les copies réalisées par Rousseau et dont le mémoire de Neufchâtel conserve la trace, comportaient peut-être quelques-uns de ces portefeuilles de musique manuscrite?

$\mathrm{Au}$ XVIII ${ }^{\mathrm{e}}$ siècle, on n'accordait pas aux autographes musicaux «utilitaires " - encore moins à ceux provenant de copistes - la valeur qu'ils revêtent aujourd'hui... On peut toutefois espérer que des copies autographes de Rousseau attendent encore d'être identifiées dans les fonds de quelques bibliothèques privées ou publiques. Ainsi la liste des Copies de la Musique faites par J.J. Rousseau pour S.A.S. Monseigneur le Prince de Conti pourrait-elle prendre une autre réalité musicale...

Thomas VERNET

Fondation Royaumont, Bibliothèque musicale François-Lang 


\section{Annexe}

Bibliothèque publique et universitaire de Neuchâtel. Fonds Rousseau, MsR-NA9 fol 4-7.

Copies de Musique

\section{Pour S.A.S. Monseigneur le Prince de Conti}

Par J.-J. Rousseau

D.

\begin{tabular}{|c|c|c|c|}
\hline 1. Più non si trovano & 9 pages à $6^{s}$ & $2^{\text {lt }} 14^{\mathrm{s}}$ & [Galuppi, L'Olimpiade] \\
\hline 2. Sprezza lo stral & 10 & & [Jommelli, Tito Manlio] \\
\hline 3. Ogni amator suppone & 8 & $2: 8$ & $\begin{array}{l}\text { [Hasse, Jommelli ou Galuppi, Didon } \\
\text { abbandonata] }\end{array}$ \\
\hline 4. Io parto...Duetto & 12 & $3: 12$ & [Ariosti] \\
\hline 5. Và, più non dirmi & $81 / 2$ & $2: 11$ & [Galuppi ou Jommelli, Ipermestra] \\
\hline 6. La bella mia & 8 & $2: 8$ & \\
\hline 7. Da mille furie & 8 & $2: 8$ & \\
\hline 8. Sento scherzar... terzetto & 19 & $5: 4$ & [Vinci, Medo] \\
\hline 9. Deh! Lasciami & 8 & $2: 8$ & \\
\hline 10. Ombra dell'idol mio & 9 & $2: 14$ & $\begin{array}{l}\text { [Fiorillo ou Piccinni, Alessandro nell'Indie / } \\
\text { Caldara, cantata] }\end{array}$ \\
\hline 11. Numi, se giusti siete & 11 & $3: 6$ & \\
\hline 12. Ei d'amor & 8 & $2: 8$ & $\begin{array}{l}\text { [Hasse, Lampugnani ou Vinci, Semira- } \\
\text { mide riconosciuta / Bonno, aria] }\end{array}$ \\
\hline 13. Non, sò se sdegno sia & 9 & $2: 14$ & \\
\hline 14. Vorrei sperare & 9 & $2: 14$ & [Jommelli, Caio Mario] \\
\hline 15. Sia pur sdegnato & 10 & 3 & [Abos, Alessandro nel Indie] \\
\hline 16. È questo il tuo figlio & 9 & $2: 14$ & \\
\hline 17. Ai rai di quel sembiante & 10 & 3 & \\
\hline 18. Empio, a tremar impara & 9 & $2: 14$ & [Galuppi, Evergete] \\
\hline 19. Colomba inamorata & 11 & $3: 6$ & [Gluck, Arsace] \\
\hline 20. Un aura di speme & 9 & $2: 14$ & \\
\hline 21. Son qual per mar turbato & 11 & $3: 6$ & [Galuppi, Vologeso] \\
\hline 22. Per lei mi nacque amore & 8 & $2: 8$ & [Hasse, La Spartana generosa] \\
\hline 23. Se tu mi vuoi & 8 & $2: 8$ & [Hasse, Cleofide ou Siroe re di Persia] \\
\hline 24. In te spero & 9 & $2: 14$ & [Galuppi, Gluck ou Hasse, Demofoonte] \\
\hline 25. Non curo l'affetto & 8 & $2: 8$ & $\begin{array}{l}\text { [De Majo, Galuppi, Gluck, Hasse, } \\
\text { Latilla, Pérez ou Traetta, Demofoonte / } \\
\text { Jommelli, aria] }\end{array}$ \\
\hline 26. Il suo leggiadro aspetto & 8 & $2: 8$ & \\
\hline 27. S’io moro & 9 & $2: 14$ & [Hasse, La Spartana generosa] \\
\hline 28. Non disperatie, nò & 8 & $2: 8$ & [Fiorillo / aria] \\
\hline 29. Pensa che tuo... Duetto & 13 & $3: 18$ & \\
\hline 30. Tu vuoi ch'io viva, Duetto & 12 & $3: 12$ & [Bertoni, Hasse ou Vinci, Artaserse] \\
\hline
\end{tabular}


E.

1. Tale è il rigor $\quad$ Recitativo 7

\begin{tabular}{lccc} 
& & 18 & $5: 8$ \\
\hline 2. Ben che veda & Aria & 9 & \\
\hline 3. Che angustia è questa & Recitativo & & \\
& 11 & $5: 14$ \\
& 19 & &
\end{tabular}

\begin{tabular}{lcc}
\hline 4. Dal Ciel sdegnato & Arira & 8 \\
\hline 5. Oh me infelice & Recitativo & 23 \\
& & 31
\end{tabular}

\begin{tabular}{|c|c|c|c|c|}
\hline \multicolumn{5}{|c|}{$9: 6$} \\
\hline 6. La Sorte mia & Aria & 8 & & [Hasse, Siroe re du Persia] \\
\hline 7. Berenice, ove sei & Recit & $\begin{array}{l}12 \\
24\end{array}$ & $7: 4$ & \\
\hline 8. Ombra che pallida & Aria & 12 & & $\begin{array}{l}\text { [Di Capua, Jommelli, ou } \\
\text { Sarti, Vologeso re de'Parti / } \\
\text { Arja ou Piccinni, aria] }\end{array}$ \\
\hline 9. Si vezzose pupillette & & 9 & $2: 14$ & $\begin{array}{l}\text { [Hasse, Cleofide / Dal Barba, } \\
\text { aria] }\end{array}$ \\
\hline 10. Quando sarà quel di & & 10 & 3 & $\begin{array}{l}\text { [Hasse, Jommelli ou Sarti, } \\
\text { La Clemenza di Tito] }\end{array}$ \\
\hline
\end{tabular}

\begin{tabular}{lccl}
\hline 11. Sgombra dall'animo & 9 & $2: 14$ & \\
\hline 12. Scherza il Nocchier & 9 & $2: 14$ & $\begin{array}{l}\text { [Arja, Hasse ou Leo, Il } \\
\text { Demetrio / Traetta, cantata] }\end{array}$ \\
\hline 13. Il mio destin & 8 & $2: 8$ & $\begin{array}{l}\text { [Leo, Scipione nelle Spagne / } \\
\text { Patoni, aria] }\end{array}$ \\
\hline 14. Lascia cadermi & 8 & $2: 8$ & [Hasse, Artaserse]
\end{tabular}

